\title{
Jürgen Hoffmann \\ Zersetzungsprodukt oder strukturierender Faktor in der Restrukturierungskrise? - Gewerkschaftspolitik in der Bundesrepublik in den 80er Jahren: ein Szenario"
}

\section{Gewerkschaftspolitik ohne soziale Bewegung - Die These von der "Amerikanisierung " der deutschen Gewerkschaftsbewegung}

Die Gewerkschaften in der Bundesrepublik haben im internationalen Vergleich die ökonomische Krisenphase seit Mitte der 70 Jahre erstaunlich unbeschadet durchgestanden: Zumindest bis zum zweiten zyklischen Einbruch der ökonomischen Entwicklung Anfang der 80er Jahre konnten - nach kurzer Stagnation 1976 - die Realeinkommen der Beschäftigten jährlich angehoben werden, der Kern der Mitgliedschaft der Industriegewerkschaften - vom Öffentlichen Dienst ganz zu schweigen - konnte vor massiven materiellen Beeinträchtigungen durch Arbeitsplatzverlust, Abgruppierungen, Dequalifikationen und Lohnabbau allgemein bewahrt werden.

Die (relative) Stabilität der deutschen Gewerkschaften ist dabei sicherlich nicht nur auf günstige äußere Bedingungen zurückzuführen (wie die auch in und nach der Weltwirtschaftskrise 1975 noch günstige Stellung der bundesdeutschen Industrie auf dem Weltmarkt), sondern auch auf die organisatorischen Formen (zentralistisch-vereinheitlichend) und die Kooperation der Gewerkschaftspolitik mit der regierenden Sozialdemokratie. Zumindest diese politische Flanke der deutschen Gewerkschaftspolitik in der Krise ist mit der »Wende« 1982 aber weggebrochen: Die konservativ-liberale Koalition sucht - unter Einschluß der Arbeitnehmer-Sozialausschüsse der CDU - nicht mehr die Kooperation, sondern die Unterordnung der Gewerkschaftspolitik unter eine gewinnorientierte Angebotspolitik im Bereich der Wirtschafts- und Sozialpolitik. Die ökonomische Basis der Gewerkschaftspolitik - auch dies schält sich in den 80er Jahren immer stärker heraus - „trägt« zwar demgegenüber noch, befindet sich aber in einem Prozeß der »Restrukturierung«, der nur vordergründig aufgrund möglicher höherer Wachstumsraten zu Hoffnungen auf einen »status quo ante« für die Gewerkschaftspolitik berechtigt. Solche Hoffnungen sind insofern trügerisch, als -- dies sei hier als später näher zu entwickelnde These vorweggenommen - sich die Bundesrepublik und der Weltmarkt der entwickelten kapitalistischen Länder seit Mitte der 70er Jahre in einer ökonomischen, gesellschaftlichen und politischen Umbruchssituation befinden, deren Charakter mit Hilfe quantifizierender Daten nicht hinreichend erfaßt werden kann: es sind nicht nur die Wachstumsraten der Ökonomie, sondern die Strukturen von Ökonomie und Gesellschaft und die Formen der Politik durch die kapitalistische Dynamik seit der Weltwirtschaftskrise 1975 zur Disposition gestellt.

Der folgende Beitrag knüpft argumentativ an früher bereits veröffentlichte Analysen zur Entwicklung der Gewerkschaftsbewegung in der Bundesrepublik an (vgl. insbes. Hoffmann 1981). Im Zentrum dieser Analysen stand die Frage nach der »Amerikanisierung« der deut-

* Vorabdruck aus Müller-Jentsch, W. (Hg.), Gewerkschaften im Umbruch - ein internationaler Vergleich (Arbeitstitel), erscheint voraussichtlich Ffm 1987 
schen Gewerkschaftsbewegung in und nach der Krise 1975. Dieser Terminus sollte die Gefahr einer branchen- und fraktionsspezifischen Auseinanderentwicklung der gewerkschaftspolitischen Interessenvertretung unter Krisenbedingungen benennen. Zusammengefaßt - und um die Dimension der »Individualisierung« in der Reproduktionssphäre erweitert - lautete die These, daß

- die ökonomische Entwicklung von außen die Erfolgsbedingungen für eine solidarische Interessenvertretungspolitik der Gewerkschaften in der Bundesrpeublik vorgab und die Vollbeschäftigungssituation die tatsächlichen Differenzen und Differenzierungen verdeckte, die erst mit der Krise und Massenarbeitslosigkeit seit 1975 wieder deutlich sichtbar und zum Problem gewerkschaftlicher Politik werden;

- daß diese vom Kapital vorgegebene Entwicklung zugleich durch einen Prozeß der »Individualisierung « in der Produktions- und Reproduktionssphäre charakterisiert ist, der die Interpretation der Situation des einzelnen in Produktion wie Reproduktion losgelöst hat von kollektiven Interpretations- und Handlungsmustern im Arbeits- und Lebensprozeß, und daß diese Erfahrung auch das Muster einer weitgehend individualistischen Kriseninterpretation und Krisenbewältigungsform in den 70er Jahren vorgab;

- daß somit die veränderten Strukturen der kapitalistischen Entwicklung seit 1975 ("Strukturbruch«, »strukturelle Überakkumulation«) die Erfolgsbedingungen gewerkschaftlicher Politik in ihr Gegenteil verkehren.

Hervorzuheben ist in diesem Zusammenhang, daß der Erfolg der Gewerkschaftspolitik aufgrund der vom Kapital produzierten Arbeitsmarktverhältnisse möglich war - ohne eine soziale Bewegung im Hintergrund: Die organisatorischen Formen und kommunikativen Strukturen der Arbeiterbewegung waren durch Faschismus, Krieg, den Strukturwandel der Nachkriegszeit und in der Prosperitätsphase weitgehend eliminiert worden, auch wenn sich die Gewerkschaftspolitik mit ihren Symbolen weiterhin schmückte. Entscheidend für Produktion wie Reproduktion war, daß die durch die »Natur« des kapitalistischen Produktionsprozesses vorgegebene Form der Fraktionierung und Differenzierung und die durch die Entwicklung der Massenkonsumstrukturen vorangetriebene »Individualisierung « (Beck) ${ }^{1}$ nicht mehr durch eine - im Faschismus zerstörte - Arbeiterbewegung und. Arbeiterkultur aufgefangen werden konnte, sondern eher noch über die kapitalistisch produzierte »Auflösung des proletarischen Milieus" (Mooser) befördert wurde. Die mit der Kapitalakkumulation gegebene Verfestigung des sozialen Herrschaftsverhältnisses in der Produktion und systematische Unterprivilegierung der lohnabhängigen Klasse in der Reproduktion (vgl. zum Letzteren Oppolzer 1986) stehen einer solchen Individualisierung zwar entgegen, werden aber von diesem Prozeß überwölbt.

Unter dem Mantel einer allgemeinen Erhöhung des Lebensstandards und Verallgemeinerung des Lohnarbeiterstatus (bei U. Beck »Homogenisierung») wurden so quasi US-amerikanische Interessenvertretungsstrukturen entwickelt. $\left.{ }^{2}\right)$ Diese fraktionsspezifischen Vertretungsstrukturen und die individualistischen Interpretations- und Handlungsmuster mußten in dem Moment als Restriktion für eine solidarische Interessenvertretung sichtbar werden, in dem die ökonomische Basis einer scheinbar erfolgreichen Gewerkschaftspolitik wegbrach und auch die seit 1967 existierende staatliche Flanke in Form der keynesianischen Globalsteuerung ihre zentrale gesellschaftspolitische Intention (die Garantie der Vollbeschäftigung) nicht mehr ausfüllen konnte. Die Krise 1975 und die nachfolgende andauernde Massenarbeitslosigkeit ist so ein erster Bruchpunkt in der gewerkschaftlichen Politik der Bundesrepublik. 
Der ökonomische Strukturbruch seit $1975^{3}$ zeigt sich an zwei - für die Gewerkschaftspolitik bedeutsamen - Phänomenen: Die tiefe Krise 1975 führt zu einer Massen- und Dauerarbeitslosigkeit von über einer Million Arbeitslosen, die bei der folgenden tieferen zyklischen Krise 1980 ff. den Sockel von über zwei Millionen Arbeitslosen erreicht. Massenarbeitslosigkeit gehört so seit 1975 zur »Normalität« des kapitalistischen - und gewerkschaftlichen Alltags in der Bundesrepublik. Darüber hinaus zeigt die Massenarbeitslosigkeit aber auch das Scheitern des politischen Versprechens an, über die keynesianische Globalsteuerung Vollbeschäftigung und Verstetigung des Einkommenszuwachs für alle Erwerbstätigen erreichen zu können. Bereits vor der »politischen« Wende 1982 deutet sich unter sozialdemokratischer Regierung ein Formwechsel der Politik an, der durch eine Umorientierung in Richtung Strukturpolitik und Einfrieren der sozialstaatlichen Ausgaben (bzw. Umbau in diesen Ausgaben, Kürzungen an den »Rändern«: Schüler, Behinderte etc.) gekennnzeichnet ist. Beide Phänomene, Massenarbeitslosigkeit und Scheitern der keynesianischen Vollbeschäftigungspolitik, zeigen einen ökonomischen Strukturbruch an, dem sich in der Folge die staatliche wie auch die gewerkschaftliche Politik anpassen muß.

\section{Korporatistische Formen der Interessenvertretung gegen (neue) soziale Bewegungen: Gewerkschaftspolitische Konstellationen nach der Krise 1975 bis zur "Wende* 1982}

Als ein Fazit der durch die Krise 1975 eingeleiteten Entwicklung der gewerkschaftspolitischen Rahmenbedingungen kann die These aufgestellt werden, daß mit der jetzt sich entwickelnden überzyklischen Massenarbeitslosigkeit, dem Scheitern des »keynesianischen Klassenkompromisses« und dem Ende des Ausbaus des Sozialstaates die herkömmliche ökonomische und politische Basis der Gewerkschaftspolitik zersetzt wird bzw. nicht mehr existiert. Ökonomisch ist die Basis für eine solidarische Interessenvertretung im Sinne einer über die Branchenunterschiede hinweg durchzusetzenden allgemeinen Lohnerhöhung bei Vollbeschäftigung nicht mehr möglich, politisch wird eine solche Politik nicht mehr abgestützt, und der Einfluß der Gewerkschaften auf die Politik wird sogar zurückgedrängt, weil deren Forderungen eher die jetzt angestrebte Politik der Haushaltskonsolidierung behindert.

Mit der Veränderung der ökonomischen Basis und der politischen »Flanke« wird aber die herkömmliche Gewerkschaftspolitik, gerade weil sie sich (vorerst) nicht verändert, bezogen auf eine solidarische Interessenvertretung in ihren Ergebnissen ambivalent bzw. sogar dysfunktional: Die durch die ökonomische Prosperität und die Vollbeschäftigung verdeckten differenzierenden Strukturen werden jetzt deutlich und zum Problem einer einheitlichen, solidarischen Gewerkschaftspolitik zwischen den Einzelgewerkschaften und in den Einzelgewerkschaften. Eine sich nur auf die ökonomischen Strukturen beziehende Gewerkschaftspolitik reflektiert so notwendigerweise die ökonomischen Differenzierungen und — darauf wird unten noch näher einzugehen sein - die ökonomischen, technologischen und personalpolitischen Strategien der Einzelkapitale. Sie wird unter diesen Bedingungen Reflex kapitalistischer Konkurrenz und nicht deren Widerpart.

\section{1. "Kern und Rand « - Fraktionierung in der Produktion als soziale Barriere gegen ein Durchschlagen der Arbeitslosigkeit auf die Teilarbeitsmärkte}

Die neue Konstellation für die Gewerkschaftspolitik nach 1975 kann stichwortartig in fünf Punkten zusammengefaßt werden: 
1. Auf Basis allgemein gesunkener Akkumulationsraten und vorherrschender Rationalisierungsinvestitionen entwickelt sich jetzt überzyklisch eine Massenarbeitslosigkeit. Damit ist aber noch nicht das für die Gewerkschaftspolitik entscheidende »Eckdatum« hinreichend bekannt. Denn während in der Prosperität eine allgemeine Knappheit der Ware Arbeitskraft bestimmend war, sind es gerade nicht - als einfacher Gegensatz dazu — das allgemeine Überangebot der Ware Arbeitskraft, sondern die auf Basis dieses »Überangebots« hervorgetretenen Teilarbeitsmärkte, die jetzt gewerkschaftspolitisch relevant werden: Sei es über die gegebenen, sich ausdifferenzierenden ökonomischen Strukturen (Branchen, Unternehmen, Abteilungen), sei es über die unterschiedlichen regionalen Arbeitsmarktbedingungen, sei es über die Formen betrieblicher Personalpolitik (und der über sie durchgesetzten Spaltung der Belegschaften in Kern- und Randbelegschaften), oder sei es durch Prozesse der »sozialen Schließung (die durch die vorgenannten Faktoren aber in aller Regel vorstrukturiert werden) - eine diese Differenzierungen überwölbende solidarische Interessenvertretung wird auf Basis des gegebenen ökonomischen $\mathrm{Be}-$ zugspunktes der Gewerkschaftspolitik nicht mehr möglich. Im Gegenteil: Möglich wird auf dieser Basis der Erfolg einer Interessenvertretung, die die Knappheit der Ware Arbeitskraft auf einzelnen Teilarbeitsmärkten ausnutzen kann bzw. durch eine Politik der Besitzstandswahrung Prozesse der sozialen Schließung befördert und lohnpolitisch differenzierend auszunutzen versteht. Und damit sind die Einzelgewerkschaften durchaus »basisnah", denn die Mitglieder der so geschützten Kernbelegschaften sind in der Regel auch der Kern der Mitgliedschaft: der »deutsche, männliche Facharbeiter mittleren Alters herrscht hier vor. Mit dem Andauern der Massenarbeitslosigkeit verfestigt sich diese Struktur sogar noch weiter, denn einmal fallen immer größere Teile der Dauerarbeitslosen ganz als "Marginalisierte aus der Arbeitsmarktkonkurrenz heraus, zum anderen konzentriert sich dieser Status der Marginalisierten auf „Problemgruppen« des Arbeitsmarktes (Jugendliche, ältere Arbeiter/innen, Frauen als "Zuverdiener«, Ausländer, Behinderte), die aufgrund ihrer jeweiligen »askriptiven Merkmale« (Offe 1977) aus der Arbeitsmarktkonkkurrenz herausgehalten werden und die nicht zum Mitgliederkern der Gewerkschaft gehören - auch wenn z.B. bei Frauen und Ausländern neuerdings der Organisationsgrad steigt.

2. Die Rationalisierungsinvestitionen in und nach der Krise 1975 , die aufgrund der gesunkenen Akkumulationsrate ( = Erweiterung der Produktion) an Gewicht gewinnen, vernichten jetzt Arbeitsplätze, und auch der Wegfall von Qualifikationen aufgrund technologischer Innovationen kann jetzt nicht mehr - wie das noch in den 60er Jahren die Regel war - durch Umsetzung auf gleichwertige Arbeitsplätze in anderen Unternehmen und Branchen kompensiert werden. So bleibt nur die (in den Fällen von Facharbeitern in Wachstumsbranchen begründete) Hoffnung, zu den Rationalisierungsgewinnern zu gehören. Auch verändern die Rationalisierungsinvestitionen unter Krisenbedingungen die Arbeitsbedingungen - ein Aspekt, auf den bei der Darstellung des Restrukturierungsprozesses in den $80 \mathrm{er}$ Jahren unten näher einzugehen ist.

3. Die Formen der Verrechtlichung der industriellen Beziehungen erweisen sich in der Krise nicht nur als Schutz vor dem Wirken der Arbeitsmarktkonkurrenz insbesondere im Entlassungsprozeß, sondern sind - insofern durchaus ambivalent - Moment der Individualisierung auf dem Arbeitsmarkt. Der »Prozeß der Arbeitslosigkeit« (Büchtemann 1984) ist gerade dadurch charakterisiert, daß über die arbeits- und sozialrechtlichen Formen der Verarbeitung das Schicksal des Arbeitslosen individualisierend durch die Arbeits- und So- 
zialbürokratie buchstäblich »klein «-gearbeitet wird. Ein kollektiver Bezug ist so auch institutionell mit anhaltender Dauer der Arbeitslosigkeit immer weniger gegeben (Arbeitsgericht - Arbeitsamt - Sozialamt).

4. Auch die vereinheitlichende Leistung des dualen Systems der Interessenvertretung erweist sich für die Einheitsgewerkschaft in der Krise als durchaus ambivalent: Einzeluntersuchungen belegen die Absicherung der Kernbelegschaften im Sinne einer sozialen SchlieBung gerade auf Betriebsratsebene, wobei Interessenkongruenzen zwischen Betriebsrat (Sicherung der Kernmitgliedschaft der Gewerkschaft) und betrieblicher Personalpolitik (Erhaltung eines leistungsfähigen Kerns der Facharbeiterschaft) bestehen und über die Mitbestimmung bei Kündigungsprozessen zum Tragen kommen.

5. Das Konzept einer »aktiven Strukturpolitik « - das von Scharpf/Hauff (1975) als sozialdemokratische Antwort auf die Strukturkrise entwickelt wurde - baut die Gewerkschaft, gerade in ihrer eingeschränkten Funktion als Interessenvertretung auf den Teilarbeitsmärkten, in einen »selektiven Korporatismus« (J. Esser) ein: Die Politik unterstützt gezielt die Wachstumsbranchen und das dort vorhandene »Humankapital« (den deutschen Facharbeiter) als Konkurrenzvorteil auf dem Weltmarkt; die Gewerkschaften müßten diesen Prozeß durch eine korporatistische Zusammenarbeit mit dem Kapital in diesen Branchen unterstützen, während das »Gesundschrumpfen« der strukturschwachen Branchen (die nicht mehr durch staatliche Subventionen gestützt werden) durch die jeweiligen Gewerkschaften legitimatorisch abgesichert würde. M.a.W.: die Funktion der ökonomischen Krise wird hier politisch antizipiert.

Zusammenfassend muß daher der Erfolg und die Stabilität der deutschen Gewerkschaften in der Krise (organisatorische Stabilität und Reallohnerhöhungen bis 1980) kritisch betrachtet werden: dieser Erfolg war möglich auf Basis des Hervortretens von Teilarbeitsmärkten, auf denen eine relative Knappheit der Ware Arbeitskraft durch Segmentierungslinien und über Prozesse der sozialen Schließung bewahrt werden konnte. Insofern war dieser »Erfolg « eher ein Reflex der tatsächlichen Schwäche der Einheitsgewerkschaft und wirkte zersetzend.

\section{2. "Spaltung der Gesellschaft« - Gewerkschaften als Interessenvertreter der Arbeitsplatzbesitzer gegen soziale Bewegungen?}

Mit diesen die Basis einer Einheitsgewerkschaft zersetzenden Krisenfolgen verliert die Gewerkschaftsbewegung aufgrund der engen Anbindung ihrer Interessen an die gegebenen ökonomischen, technologischen und politischen Strukturen auch an gesellschaftlicher Hegemonie. Die unkritische Übernahme der wachstumspolitischen Imperative, die hohe Akzeptanz technologischer Innovationen als Basis von zukünftigen Reallohnsteigerungen (bei sozialstaatlicher Absicherung des individuellen Risikos) und die enge ideologische und politische Verflechtung mit der sozialdemokratischen, »etatistischen« Politik machten die Gewerkschaften hilflos gegenüber der sich seit Beginn der 70er Jahre entwickelnden Kritik von (»neuen«) sozialen Bewegungen. Diese kritisieren die ökologischen Auswirkungen des konkurrenzhaft sich durchsetzenden Wirtschaftswachstums, die Gefährdungen durch die Großtechnologien, die undemokratischen korporatistischen Verbindungen zwischen Staatsapparaten und Interessenverbänden und die die inneren Reformen begleitenden bürokratischen Strukturen (»soziale Reformen von oben«). Indem diese Kritik sich an den Resultaten kapitalistischer Akkumulation festmacht, kritisiert sie zugleich kapitalistisch fixierte Interessenstrukturen, in die die Gewerkschaften eingebunden sind und auf die sie sich positiv beziehen 
müssen: Die Fixierung von Kapital z.B. in der Atomenergiebranche ist eben gleichbedeutend mit der Fixierung von Interessen der dort beschäftigten Lohnarbeiter und der sie vertretenden Gewerkschaften. ${ }^{4}$

Diese Situation spitzt sich aber mit der anhaltenden und in der Krise 1980-1982 sich verdoppelnden Massenarbeitslosigkeit zu: Die Gesellschaft spaltet sich zusehends in einen Bereich der "Einkommensquellenbesitzer (Kapital-Profit, Arbeitsplatz-Lohn etc.) und einen marginalisierten Bereich der Dauerarbeitslosen, der zugleich einen wachsenden Anteil von »neuen Armen« aufweist. Mit der sich herausbildenden »neuen Armut« wird zugleich deutlich, daß die nicht zuletzt von der Arbeiterbewegung erkämpften kollektiven und sozialstaatlichen Absicherungen in Phasen der andauernden Massenarbeitslosigkeit nicht mehr greifen, weil sie an den Beschäftigtenstatus gebunden sind: Die Spaltung der Gesellschaft stellt so immer deutlicher auch Ansatzpunkte traditioneller Arbeiterpolitik in Frage, Problemdimensionen, die jetzt soziale Bewegungen (z.B. Arbeitsloseninitiativen und die "Grünen«) gegen die traditionellen Formen der Arbeiterpolitik aufgreifen (vgl. Leibfried/Tennstedt 1985). Das ist um so wichtiger, als ja (anders als in der Weimarer Republik) die Entlassungen nicht mehr durch ein funktionierendes Arbeitermilieu aufgefangen und politisch integriert werden: Da die Gewerkschaftspolitik die Arbeitslosen der individualisierenden staatlichen Fürsorge überläßt, begibt sie sich der Möglichkeit, eine soziale Bewegung als kollektive Interpretations- und Politikform den Arbeitslosen anzubieten und sich selbst diese Flanke freizuhalten.

Wenn oben die Phase der Vollbeschäftigung als eine Phase erfolgreicher Gewerkschaftspolitik ohne soziale Bewegung (hier: Arbeiterbewegung) gekennzeichnet wurde, dann muß die Entwicklung seit dem krisenhaften Bruch Mitte der 70er Jahre zunächst als eine Phase der Reduktion gewerkschaftlicher Politik auf die konkurrenzhaft vorgegebene Situation der Branchenkapitale gegen die entstehenden neuen sozialen Bewegungen umschrieben werden. Während so auf der einen Seite die Gewerkschaften funktional sich den Interessenvertretungsformen eines »ADAC (Müller-Jentsch 1984) annähern, werden die gesellschaftlichen Themen nach 1975 von neuen sozialen Bewegungen und dem Neokonservatismus besetzt, der die Systemimperative der privat-dezentralen kapitalistischen Ökonomie radikal ins Politische übersetzt.

Die Ursachen des Erfolgs neokonservativer Ideologie liegen nicht zuletzt in den Erfahrungen der einzelnen Lohnabhängigen mit Prosperität und Krise begründet: So wie die Konkurrenz der Kapitale um die knappe Ware Arbeitskraft in der Prosperität der eigenen Leistung zugeschrieben wurde, so wird jetzt die Erfahrung der Arbeitslosigkeit auf das individuelle Versagen zurückgeführt - eine Interpretation, die durch die Selektion der betrieblichen Personalpolitik bei Entlassungsprozessen Bestätigung findet. Da zugleich eine kollektive Kriseninterpretation und -bewältigungsstrategie fehlt (die Gewerkschaften können nach 1975 zunächst nur die verblassenden Hoffnungen auf die staatliche Wirtschaftspolitik anbieten) und eine soziale Bewegung nicht mehr als Arbeiterbewegung existiert (die früher beides anbieten konnte), werden hier Einbruchstellen für eine individualistische Leistungsideologie aufgetan, die die Neokonservativen in CDU/CSU und FDP seit Mitte der 70er Jahre ausnutzen (vgl. auch Schumann 1979).

Auch in dieser Perspektive erweist sich der relative Erfolg der gewerkschaftlichen Politik in der Krise und bei Massenarbeitslosigkeit als ein »Pyrrhus-Sieg«: die Stabilität der Organisation, die Erhöhung der Realeinkommen für die Beschäftigten bis 1980 (auf Basis der Verknappung auf Teilarbeitsmärkten), die Erhöhung des Organisationsgrades der DGB-Gewerkschaften - diese relativen Erfolge der Gewerkschaftspolitik in der Krise verdecken den 
grundlegend gewandelten Charakter der Basis gewerkschaftlicher Politik nach 1975. Denn diese Basis ist in der Bundesrepublik in besonderer, historisch begründeter Weise ökonomisch determiniert und seit 1975 in einen Prozeß der Restrukturierung einbezogen, der in einer langfristigen Perspektive als $»$ Formkrise (Altvater 1983) bezeichnet werden kann und in unterschiedlichen wissenschaftlichen Paradigmata (»Lange Wellen«, »Fordismus-Theorie«, »Strukturelle Überakkumulation «) übereinstimmend als umfassender Prozeß der ökonomischen, sozialen und politischen Restrukturierung begriffen wird. Die Konturen dieses Prozesses werden dabei erst in den 80er Jahren mit der politischen. Wende und den sektoralen und technologischen Restrukturierungen in und nach der Krise 1980 ff. deutlicher sichtbar.

\section{Gewerkschaftspolitik in der Restrukturierungskrise: Zersetzungsprodukt oder strukturierender Falktor?}

Ökonomische und soziale Elemente der Restrukturierungskrise wurden oben schon bei Darstellung der Wirkungen der Krise 1975 benannt. Es kommt nun darauf an, den Zusammenhang von ökonomischen, technologischen, sozialen und politischen Formen der Restrukturierung als »Krise einer historischen Struktur der Reproduktion und Regulation* zu entwickeln. Ausgehend von dieser Analyse der Rahmenbedingungen soll dann ein »Korridor « möglicher gewerkschaftspolitischer Optionen diskutiert werden.

Genau genommen muß eine Analyse des gegenwärtigen Umbruchs mit den Auswirkungen der Welt wirtschaftskrise der 30er Jahre anfangen: In der Folge dieser Krise und beschleunigt durch die Kriegswirtschaft wurden die den Nachkriegsaufschwung auf dem Weltmarkt tragenden Elemente entwickelt: (1.) der Reproduktions- und Akkumulationszusammenhang von Massenproduktion und Massenkonsum (»Fordismus«, vgl. Hirsch/Roth 1980), (2.) die technologische und arbcitsorganaisatotorische Uninstellung in der Produktion auf Basis von Fließfertigung und tayloristischer Durchdringung der Arbeitsorganisation, (3.) die Verschiebung des relativen Gewichts der einzelnen Branchenkapitale zugunsten der jetzt sich endgültig durchsetzenden Branchen der Elektro- und Maschinenbauindustrie, des Straßenfahrzeugbaus und der Chemie, die eng mit einer Welle von Basisinnovationen in diesen Branchen verbunden war (vgl. Mensch 1975, Kleinknecht 1984a und b), (4.) die Anerkennung der Gewerkschaften als Vertretungsorgan der sich jetzt entwickelnden Massenarbeiterschaft und (5.) deren Einbau in ein System staatlicher Regulierung des entwickelten Kapitalismus, das auf der Basis keynesianischer Politikansätze sich in unterschiedlichen Formen vor und nach dem zweiten Weltkrieg entwickelt (New Deal in den USA, faschistische Wirtschaftsregulierung in Deutschland, Keynesianismus der Nachkriegszeit). In diesem »Modell« gesellschaftlich-kapitalistischer Reproduktion warer die Gewerkschaften ein Stützpfeiler seines Funktionierens: die zunächst hohen Produktivitäten erlaubten eine erfolgreiche produktivitätsorientierte Lohnpolitik, die wiederum den Absatz der Massenkonsumgüter ermöglichte und die Arbeiterschaft organisatorisch in das Modell staatlicher Regulierung integrierte, dabei den Gewerkschaften aber zugleich eine solidarische Lohnpolitik ermöglichte: "keynesianischer Klassenkompromiß« (Vgl. Buci-Glucksmann 1982, Hoffmann 1982).

Die zentralen Elemente der sich über die Krisen 1975 und 1980-82 herausbildenden neuen Struktur der ökonomischen, sozialen und politischen Reproduktion stehen teilweise diametral gegen diese Wachstumsstruktur der Nachkriegszeit. Entscheidend für die sich herausbildenden Strukturen sind nämlich: 
- die seit der Krise 1975 vorgegebene offene Fraktionierung in der Produktion und Spaltung in der Gesellschaft und die darauf aufbauende Individualisierung und Konkurrenz;

- der Bedeutungsverlust klassischer Industriebranchen mit vorherrschender Massenproduktion und hohem gewerkschaftlichen Organisationsgrad (Eisen- und Stahlindustrie, Schiffbau; allgemeiner: Grundstoff- und Produktionsgüterindustrien und Bergbau), der Rückgang arbeitsintensiver Bereiche der Konsumgüterindustrien (Bekleidungsgewerbe, Textilindustrie) und der Bauindustrie;

- die technologische Umwälzung auf Basis der Halbleitertechnologie und der darauf aufbauenden Entwicklung von Steuerungs-, Sensor- und Verkettungstechnologien, durch die es möglich wird, die Produktivität in der Kleinserien- und Einzelfertigung zu erhöhen, die Massenfertigung zu flexibilisieren, Facharbeiterqualifikationen zu ersetzen bzw. auf eine neue Stufe zu heben (»neue Produktionskonzepte«), die eine »Autonomie« der Facharbeit für einzelne ermöglichen; zugleich Kontrolle der Arbeitsabläufe durch das Management über neue Kommunikationstechnologien;

- die über die neuen Informations- und Kommunikationstechnologien möglichen Formen der Rationalisierung der privaten und öffentlichen Verwaltungstätigkeiten (der »toten Kosten« der Produktion), der Dezentralisierung der Produktion und damit der optimalen, flexiblen Ausnutzung der Teilarbeitsmärkte, die tendenziell mit der Entwicklung neuer Formen der Lohnarbeit im Sinne eines »Sub-Unternehmerstatus« verbunden werden können;

- eine Umstrukturierung in der industriellen Produktion zugunsten der Produktion dieser neuen Technologien (zunächst in dezentralisierten Produktionseinheiten);

- eine relative Verschiebung der Beschäftigungsanteile in Richtung "Dienstleistungssektor«, der aufgrund seiner Strukturen einer gewerkschaftlichen Organisierung große Hindernisse in den Wert legt ${ }^{7}$;

- und - als entscheidende Voraussetzung und Rahmenbedingung - der Formwandel der Politik, die jetzt gegen die Fixierung sozialer Besitzstände (in der Form der Fixierung von Kompromißlinien zwischen Lohnarbeit und Kapital) die arbeits- und sozialrechtlichen Voraussetzungen und Rahmenbedingungen einer individualisierten und flexiblen Produktion schaffen und erhalten soll. Es geht daher um den »Umbau « (nicht nur um den Abbau) des Sozialstaats (vgl. Altvater 1982).

Dieser Prozeß der Restrukturierung ist nicht "gradlinig« mit der Krise 1975 umgesetzt worden. Vielmehr könen wir zwei Phasen festhalten, die als Phasen der Restrukturierungskrise dargestellt werden können: (1.) Die Phase der Kapitalvernichtung und -entwertung in den beiden tiefen zyklischen Krisen 1975 und 1980-82, in denen Teile der bislang die Akumulation tragenden Industrien durch Kapitalentwertung umstrukturiert wurden, und (2.) die Entwicklung nach 1980/82, in der neue Techniklinien im Zuge einer wieder sich beschleunigenden Akkumulation eingesetzt werden. Dieser Prozeß dürfte aber noch über einen Zeitraum von 10 bis 15 Jahren andauern und zugleich auch eine Phase des Experimentierens und des Suchens nach optimalen Faktorallokationen auf Basis der neu entwickelten Technologien sein, der ökonomisch auch von Kapitalentwertungen und Konzentrationsprozessen in den diese neuen Techniklinien produzierenden und anwendenden Industrien begleitet wird. Die industriellen Beziehungen erfahren durch die neuen Formen des Zusammenhangs von Ökonomie, Technologie und Politik eine entscheidende Veränderung: In wichtigen aktuellen Untersuchungen wird bei allen Unterschieden im Detail immer wieder der Zusammenhang dieser neuen Formen gesellschaftlicher Produktion als »flexibler Spezialisierung" als 
Kern des Restrukturierungsprozesses herausgestellt (vgl. Baethge/Oberbeck 1986, S. 23; Kern/Schumann 1984, S. 48).

Auch wenn sich diese Tendenzen nicht bruchlos verallgemeinern und in die Zukunft verlängern lassen ${ }^{8}$, so wird doch bereits ein »Korridor « zukünftiger Entwicklungen in den industriellen Beziehungen deutlich:

(1.)Zunächst ist gegenüber einer euphorischen Sicht des neuen Rationalisierungstyps und des daraus entspringenden Typus von Facharbeit (vgl. zu einer solchen Euphorie Glotz 1986) festzuhalten, daß (a) hier nur bestimmte Bereiche der Industrie (bei Kern/Schumann: die Wachstumsindustrien) und der Dienstleistungen (bei Baethge/Oberbeck: Handel und Verwaltung) untersucht wurden und (b) nur die »Rationalisierungsgewinner — - so die These - von dem neuen, ganzheitlichen Zuschnitt der Facharbeit profitieren.

Resultat ist demnach, daß in beiden Bereichen verstärkt Arbeitskräfte freigesetzt werden und der Bereich »Büro und Verwaltung« auch keine Kompensationsfunktionen mehr für die Freigesetzten des primären und sekundären Sektors übernehmen wird. Die dominante Form der Dequalifikation wird daher der Ausschluß aus der Arbeit sein (vgl. Baethge/Oberbeck 1986, S. 32). Offen bleibt allerdings, inwiefern andere, arbeitsintensive Bereiche des Dienstleistungssektors expandieren und zusätzliche Arbeitsplätze im Bereich der »bad jobs«, z.B. bei »McDonalds«..., schaffen (vgl. dazu Schmiede/v. Greiff 1985, S. 293).

Die mit der Freisetzung gegebene Möglichkeit einer verschärften Konkurrenz auf dem Arbeitsmarkt und des Durchschlagens dieser Konkurrenz auch auf die $\gg$ Rationalisierungsgewinner (vgl. auch Schmidt 1985) kann schon deshalb nicht ausgeschlossen werden, weil - so z.B. Baethge/Oberbeck - die Ergebnisse der »systemischen Rationalisierung« erst im nächsten Jahrzehnt voll auf das Beschäftigungssystem durchschlagen werden (S. 39). Das Auffüllen der industriellen Reservearmee durch die »Rationalisierungsverlierer « ist dabei auch insofern von Bedeutung, als offensichtlich die »neuen Produktionskonzepte« die Wiedereingliederungsbarrieren für Dauerarbeitslose erhöhen und so zugleich die Bindung an den Betrieb bei den Beschäftigten verfestigen. (Vgl. dazu Kern/Schumann 1984, S. 309 ff., Baethge/Oberbeck 1986, S. 40 ff.)

(2.) Die Wiedergewinnung von Autonomie und umfassender Qualifikation muß nicht allein zu einem - gewerkschaftspolitisch aufzugreifenden - Prozeß der Selbstbestimmung und der Persönlichkeitsentwicklung führen, sondern ist zunächst einmal mit einem weitergehenden Prozeß der Individualisierung verbunden und kann - worauf die hier diskutierten Studien auch hinweisen - zu korporatistischen bzw. neosyndikalistischen Interessenvertertungsstrukturen führen. Solche Entwicklungen liegen gerade deshalb nahe, weil der Druck der industriellen Reservearmee dafür sorgen wird, die Bindung der Rationialisierungsgewinner an den Betrieb zu erhöhen und Prozesse der sozialen Schließung zu befördern. Auch bleibt noch abzuwarten, ob unter diesem Druck nicht neue Formen der Intensivierung der Arbeit möglich sind.

(3.) Denn die neuen Rationalisierungskonzepte - das wird aus allen Studien deutlich, vgl. aber auch Kubicek/Rolf 1985 - sind aufgrund des Systemcharakters der eingesetzten Technologie auch eine neue Stufe der Kontrolle nicht nur des Arbeitsergebnisses und der Arbeitszeit, sondern auch und gerade des Arbeitsablaufs(vgl. explizit Baethge/Oberbeck 1986, S. 37). Da zugleich bei den einfachen Tätigkeiten - insbesondere im Verwaltungsbereich, dem Handel und im öffentlichen Dienst - soziale durch technische Kommunikation allmählich ersetzt wird, wird durch die Vertiefung der Segmentierungslinien im 
Betrieb und durch die Veränderungen in den gewachsenen Kommunikationsstrukturen die Basis der Gewerkschaften im Betrieb reduziert, während sich die betrieblichen Herrschaftsverhältnisse auch gegenüber qualifizierten Arbeitergruppen in Richtung auf mehr "laufende« Kontrolle verändern.

(4.) Diese Formen einer »Akzentuierung « betrieblicher Herrschaftsverhältnisse« werden durch die Ausweitung der »industriellen Reservearmee« als Ergebnis des Zusammentreffens der Anwendung von Rationalisierungstechnologien, demographischer Entwicklung, nicht hinreichend beschleunigtem ökonomischen Wachstum und durch den Ausfall der sektoralen Auffangmechanismen des tertiären Sektors befördert. Selbst optimistische Varianten zur ökonomischen Entwicklung gehen von Arbeitslosenzahlen bis zum Jahre 2000 aus, die im Durchschnitt zwischen zwei und drei Millionen liegen. (Vgl. Klauder u.a. 1985, S. 59). Eine Abschottung der Beschäftigung in den betrieblichen Kernsektoren dürfte so zu einer stärkeren Anbindung an den Betrieb führen und damit die gewerkschaftspolitischen Chancen, die in den »neuen Produktionskonzepten« liegen (vgl. dazu Kern/Schumann 1984, S. 320 ff.) relativieren; darüber hinaus sind diese Chancen nur dann realistische, wenn die Restrukt urierung der Verhältnisse auf dem Arbeitsmarkt nicht auf die betriebliche Ebene durchschlägt.

(5.) Die neuen Technologien der »flexiblen Spezialisierung« machen nämlich auch eine Flexibilisierung der Arbeitsmarktstrukturen möglich und - unter betriebswirtschaftlicher Konkurrenzperspektive - ökonomisch notwendig: Aufgrund der Flexibilisierung der Produktion bringt eine Auflösung des Normalarbeitsverhältnisses (Arbeitszeitfixierung, Kündigungsschutz etc. - vgl, dazu Mückenberger 1985a) im Sinne einer Flexibilisierung des Beschäftigungsverhältnisses ökonomisch erhebliche Konkurrenzvorteile. 'Formen der Teilzeitarbeit, Leiharbeit, des »Sub-Unternehmertums« und u.U. sogar der Heimarbeit sind Instrumente der Unternehmen, die Kernbelegschaften zu vermindern und die Beschäftigung flexibel der Flexibilisierung der Produktion bei höherer Intensität der Arbeit anzupassen (vgl. dazu Dombois/Osterland 1982). Dabei knüpfen diese Beschäftigungsangebote durchaus auch an die individuellen Möglichkeiten und Wünsche der Lohnabhängigen an - wobei die "Wünscher bereits durch den Rahmen der individuellen Möglichkeiten präformiert sind (vgl. Frauenteilzeitarbeit). Diese Möglichkeiten der Flexibilisierung werden dann eine neue Stufe der Ent wicklung erreichen, wenn über die Verkabelung die Voraussetzungen einer Dezentralisierung der Produktionseinheiten und -stätten (bis in den privaten Haushalt hinein) gegeben sind und - dies ist für den Bereich Handel wichtig - Tätigkeiten auf den Kunden übertragen werden können.

(6.) Die Politik der konservativ-liberalen Regierung unterstützt diesen Restrukturierungsprozeß gezielt auf drei Ebenen: (1.) Es werden durch eine steuerliche und haushaltsmäßige Umverteilung und durch die Flexibilisierung der Arbeitsmarktverhältnisse die Nettoprofitraten erhöht, und es werden so die Möglichkeiten, die Umstellung der Produktion zu finanzieren und der Anreiz dazu, dies auch trotz hoher Zinssätze auf dem Weltmarkt zu tun, verbessert. (2.) Durch die Technologiepolitik, die von der Humanisierungspolitik weitgehend »entlastet « wird, werden die Voraussetzungen auf dem Gebiet der Grundlagen - und der angewandten Forschung für den forcierten Einsatz der neuen Technologien geschaffen (vgl. dazu Väth 1984, Hoffmann 1985); durch die Verkabelung (ISDN und BIGFCN-Systeme, vgl. dazu Kubicek/Rolf 1985) wird die entscheidende infrastrukturelle Voraussetzung für ein »Durchschlagen« der Wirkungen der »neuen Technologien « auf den Arbeitsmarkt und die industriellen Beziehungen »in den Boden verlegt«。 
(3.) Durch die Politik der »Deregulierung« soll der ordnungspolitische Rahmen für Flexibilisierungsstrategien der Unternehmen geschaffen und eine verschärfte Leistungskonkurrenz auf dem Arbeitsmarkt ermöglicht werden. Die Gesetze bzw. Gesetzesinitiativen (Jugendarbeitsschutz, Beschäftigungsförderungsgesetz, Schwerbehindertengesetz, Arbeitszeitgesetz) haben durchgehend zum Ergebnis: a) eine Förderung der betriebswirtschaftlichen Rentabilität der Einzelunternehmen, b) eine Flexibilisierung und Individualisierung der Arbeitsverhältnisse gegen die »historisch errungenen 'Normalisierungen' und Verstetigungen in der Lohnarbeiterexistenz« (Mückenberger 1985b, S. 269) und c) insgesamt eine Veränderung der industriellen Beziehungen in der Bundesrepublik: »Dort stehen nicht mehr die, in sich relativ festgefügten und strukturierten, gegensätzlichen Blöcke der Organisationen von Arbeit und Kapital einander gegenüber...; vielmehr wird die soziale Frage nunmehr vielfältig dezentralisiert und einzelfallorientiert angegangen.« (Mückenberger 1985b, S. 269). Diese Einzelfallorientierung und Individualisierung ist auch die Basis von weitergehenden ideologischen Konzepten der Neokonservativen, die das (geschichtete) Individuum zum Ausgangspunkt ihrer »Versöhnungsgesellschaft« machen (vgl. dazu Späth 1985). Damit knüpfen die Neokonservativen aber nicht nur an die neoliberale Theorie (Mückenberger 1985b, S. 255), sondern auch an technologische Möglichkeiten und das Bewußtsein des Individuums als Eigentümer seiner Arbeitskraft an: Allein aus dieser Verknüpfung kann die neokonservative Hegemonie in den 80er Jahren erklärt werden, in der ein »Aussitzen« von aktuellen politischen Problemen (Kohl) eine mehrheitsfähige Politik sein kann.

Der Politik kommt deshalb im Zusammenhang des Restrukturierungsprozesses ein besonderer Stellenwert zu, weil die ökonomischen und technologischen Restrukturierungsprozesse über die Weltmarktkonkurrrenz marktmäßig erzwungen werden und aufgrund der aufgezeigten Anbindung der Belegschaften an die einzelunternehmerischen Strategien auch sozial durchgesetzt werden können. Dagegen bilden die sozialen und politischen Resultate'der sozialen Auseinandersetzungen der vergangenen Akkumulationsphasen in Form gesetzlich und organisatorisch fixierter Kompromißlinien (vgl. dazu Blanke 1977) einen Hemmschuh für die Flexibilisierungs- und Individualisierungsstrategien der Kapitale, und das gegebene System der sozialen Sicherung verhindert eine von großen Teilen des Kapitals geforderte Lohndifferenzierung nach unten. Insgesamt steht so gerade an diesen politischen Flanken das gesamte System der »industriellen Beziehungen« zur Disposition (vgl. Bericht 1983, Späth 1984), und das Ergebnis der Auseinandersetzungen darum dürfte auch von entscheidender Bedeutung für die Ausnutzung von Chancen sein, die durch die »neuen Produktionskonzepte« im Betrieb gegeben sind.

\subsection{Die »traditionelle« Antwort der Gewerkschaftspolitik: Konservierung der sozialen} Besitzstände - Abwebr des Sozialabbaus

Diesen essentiellen Angriffen und Bedrohungen stehen die Gewerkschaften auf gesamtwirtschaftlicher Ebene ohne Bündnispartner gegenüber: Das Bündnis von sozialliberal regiertem Staat, exportorientierten Wachtumskapitalen und Gewerkschaften, auf dem das Konzept der Globalsteuerung beruhte, wird in der Krise aufgekündigt, und die Gewerkschaften geraten schon vor der politischen "Wende 1982 - zusehends in eine gesellschaftliche Isolierung. Spätestens mit der Krise 1980 ff. geraten sie ins »Fadenkreuz der Politik auch der großen Kapitale und Unternehmerverbände; allerdings wird der politische Angriff, der von der konser- 
vativ-liberalen Regierung seit 1982 umgesetzt wird, wesentlich von den mittelständischen Kapitalen geführt: Bei diesen Unternehmen können die Lohnnebenkosten (»Soziallasten«) nicht ohne weiteres durch Produktivitätserhöhungen aufgefangen werden; sie können Arbeitsmarktargumente (Druck der $\gg S c h w a r z a r b e i \ll)$ und den Hinweis auf ihre Ausbildungskapazitäten ins Feld der öffentlichen Diskussion bringen. Durchaus anzuzweifeln ist zwar, ob das Großkapital den Angriff auf die Gewerkschaften, der in der erzwungenen Veränderung des $\$ 116 \mathrm{AFG}$ deutlich wird, verstärken wird - denn gerade auf Betriebsebene sind die gewerkschaftlich garantierten sozialen Strukturen ein viel zu gewichtiges Element der Kontinuität angesichts hoher Fixkosten, so daß eine "Japanisierung « im Bereich der großen Kapitale nicht unmittelbar anstehen dürfte ${ }^{9}$. Auf gesamtwirtschaftlicher und politischer Ebene besteht für diese Kooperation aber unter den gegebenen gesellschaftlichen Strukturen kein Bedarf. Für die Gewerkschaftspolitik bedeutet der Prozeß der Restrukturierung über die schon unmittelbar in und nach der Krise 1975 erfahrenen Probleme hinaus zunächst einmal, daß auch Teile ihrer Klientele in den Wachstumsbranchen von Entlassungen bzw. Qualifikationsverlust bedroht sind, ohne daß ökonomische Kompensationen (Ausweitung des Staats-bzw. privaten Dienstleistungsektors) oder politische Unterstützung gegen diesen Prozeß zu erwarten sind. Diese unmittelbaren Auswirkungen, die durch den Abbau der gesetzlichen Schutzrechte forciert werden, stehen auch im Zentrum einer gewerkschaftlichen Defensivposition nach der »Wende« 1982, die darüber hinaus noch durch die Forderung nach nachfrage- und beschäftigungswirksamen Staatsausgaben flankiert wird und den Abbau der sozialstaatlichen Leistungen verhindern will. Soweit die technologische Entwicklung von den Gewerkschaften in eine solche Defensivposition eingebaut wird, geschieht dies in Form der Forderung nach einer »sozialen Einbindung « technologischer Entwicklung, ohne daß daraus konkrete Forderungen resultierten.

Lohnpolitisch ergibt sich aus dem ökonomischen (einzelwirtschaftlichen und branchenmäßjgen) Bezugspunkt weiterhin das zentrale Problem, daß die Verteidigung der Realeinkommen auf Basis der sich ausdifferenzierenden Verteilungsspielräume auch die materielle Situation der Lohnabhängigen ausdifferenziert und diese Entwicklung durch die politischen Rahmenbedingungen (Flexibilisierung des Arbeitsmarktes durch das »Beschäftigungsförderungsgesetz«, die Arbeitszeitgesetzgebung, auch durch die Novellierung des $\$ 116 \mathrm{AFG}$ ) enorm befördert wird. Zugleich entfernen sich Lebenslagen und Interessen von Arbeitslosen und Beschäftigten immer mehr. Und wenn sich durch eine von den Konservativen politisch angestrebte Lohndifferenzierung nach unten diese Interessen wieder »objektiv« annähern, dann sicherlich nicht in einer gewerkschaftspolitisch umsetzbaren Perspektive - die zum Ziel haben müßte, die Lebenslagen nach oben hin anzugleichen. Deutlich wird aus diesem Zusammenhang heraus aber auch, daß bereits die bloße Verteidigung der Lohnstruktur und des sozialstaatlichen Niveaus (des »Soziallohns«) gegen die Sparpolitik der konservativ-liberalen Regierung nicht nur ein Kampf um Lohnbestandteile ist, sondern - zumindest implizit - auch eine Auseinandersetzung um die untere Grenze der Lohnsätze in Form von Arbeitslosengeld/ -hilfe und Sozialhilfe. Denn der Angriff der Neokonservativen richtet sich hier wesentlich gegen die Untergrenze einer Lohndifferenzierung. Die Auseinandersetzung um Leistungen gegenüber Arbeitslosen ist somit ein Teil des Lohnkampfes - und genau hier liegen vereinheitlichende Inhalte einer auch traditionellen defensiven Lohnpolitik in der Krise (vgl. Bäcker 1985).

Das Dilemma der hier skizzierten Defensivposition als eines vorherrschenden Moments der Gewerkschaftspolitik in den 80er Jahren liegt aber in eben diesem herkömmlichen Bezugs- 
punkt: Die gegebenen ökonomischen und technologischen Strukturen des Kapitals werden als "sozialer Besitzstand « der Lohnabhängigen gegen die Dynamik der kapitalistischen Konkurrenz verteidigt, die Entwicklung der Technologie wird als Voraussetzung künftiger Verteilungsspielräume nicht kritisch hinterfragt, sondern ihre Auswirkungen sollen sozial abgefedert werden. Überspitzt könnte daher argumentiert werden, daß die Gewerkschaften die alten kapitalistischen Strukturen als Bastionen ihrer Organisations- und Konfliktfähigkeit bewahren und verteidigen wollen. Aber auch da, wo sie - wie in den Wachstumsbranchen den technischen Fortschritt als Chance der Sicherung der Arbeitsplätze durch Konkurrenzfähigkeit auf dem Weltmarkt einschätzen, bleibt die Politik den Investitionsentscheidungen der einzelnen Unternehmen verhaftet, die als fixes Datum in die gewerkschaftliche Politik der sozialen Absicherung der betroffenen Arbeitergruppen eingehen und zu »neosyndikalistischen« oder korporatistischen Vertretungsformen führen.

Diese eher traditionelle gewerkschaftspolitische Antwort auf die Restrukturierungsprozesse trägt implizit fünf Risiken in sich:

(1) Die tatsächlichen Risiken der Restrukturierung für die gewerkschaft liche Organisationsund Konfliktfähigkeit werden nicht angegangen. Die durch die neuen Technologien vorangetriebenen und durch die neuen Formen der Arbeitsorganisation verstärkten Formen der Individualisierung, Kontrolle und die Zerstörung der sozialen Kommunikation in Betrieb und Verwaltung durch die neuen Informations- und Kommunikationstechnologien werden passiv, ohne ein Konzept einer erneuerten solidarischen Interessenvertretung, hingenommen - während politisch auf eine Wahlniederlage der konservativ-liberalen Regierung gesetzt wird.

(2) Diese Politik baut auf eine beschleunigte Akkumulation, durch die aber realistischerweise weder die Ausweitung des industriellen noch des im Dienstleistungssektors enthaltenen Beschäftigungspotentials zu erwarten ist - während eine politisch zu setzende »Sozialisierung der Anpassungslasten «(Kern/Schumann) der Restrukturierung nicht nur politisch, sondern auch gesellschaftlich z.Zt. kaum durchgesetzt werden kann.

(3) Mit den Akkumulationsentscheidungen des Kapitals in der Restrukturierungsphase, der Anlage von Kapital in bestimmten Techniklinien und neuen Branchen (Elektronik, Gentechnologie, Dienstleistungsbranchen etc.) werden Interessenstrukturen für die Zukunft festgeschrieben, deren Auswirkungen auf die zukünftige Organisationsfähigkeit in der gegebenen Form mit Sicherheit negativ sein werden. Diese Akkumulationsentscheidungen sind aber - einmal in Beton, Technologie, Kabelsysteme und »soft ware « gegossen nur unter Inkaufnahme der Vernichtung von großen Massen produktiven Kapitals und darin eingebundener Arbeitsplätze umkehrbar. So werden heute die zukünftigen Interessenstrukturen festgeschrieben, die eine solidarische Gewerkschaftspolitik in der Zukunft unmöglich machen.

(4) Mit der Anbindung an die gegebenen Kapital- und Technikstrukturen muß sich diese Form der Gewerkschaftspolitik notwendig in Gegensatz bringen zu sozialen Bewegungen, die diese Strukturen besonders unter ökologischen Kriterien bekämpfen. Diese Politikform ist in der gesellschaftlichen Auseinandersetzung in einer Front mit dem Kapital und begibt sich der Möglichkeit, zusammen mit gesellschaftlichen Bewegungen die gegebene soziale Form des Wirtschaftens und ihre ökonomischen und ökologischen Resultate in Frage zu stellen.

(5) Die passive Hinnahme der technologischen Entwicklung muß -- angesichts des Stellenwerts der Verkabelung und ihrer Auswirkung auf das Freizeitverhalten - den Gewerk- 
schaften die Möglichkeit verbauen, die fortgesetzte Durchkapitalisierung des Freizeitbereichs mit ihren individualisierenden, Kommunikation erschwerenden Folgen zurückzudrängen und eigene Strukturen eines erneuerten solidarischen Milieus zu ent wickeln.

Der letztgenannte Punkt dürfte auch deshalb von Bedeutung sein, weil ein wesentliches Moment der Überwindung dieser traditionellen gewerkschaftspolitischen Antwort in den 80er Jahren in der offensiven Wiederaufnahme der Arbeitszeitverkürzungspolitik als Element einer gewerkschaftlichen Arbeitsmarktpolitik gesehen wird - deren Ergebnisse natürlich die Bedeutung der Nicht-Arbeitszeit erhöhen werden. Insgesamt aber hat die hier skizzierte "traditionelle" Antwort der Gewerkschaftspolitik kein Konzept gegen die neokonservative Hegemonie, gegen die auf individuelle Leistung und auf flexibilisierte Produktion (und Arbeitszeit) setzende Ideologie, die sich radikal zu den »Sachzwängen« der konkurrenzmäßig organisierten und auf dem Privateigentum aufbauenden Gesellschaftsordnung bekennt. Angesichts der kapitalistischen Dynamik muß diese Verteidigungslinie als »konservativ«(Dahrendorf 1984) gelten: auf die Massenarbeitslosigkeit und auf die ökologische Krise kennt sie nur die traditionellen Antworten der »Arbeiterpolitik « und des »Etatismus«, auf neue, inhaltliche Antworten verzichtet sie. Diese Politik, so wichtig sie kurzfristig als Defensivposition für die unmittelbar von Stillegungen und Rationalisierungen betroffenen Lohnabhängigen ist, kann daher kein gesamtgesellschaftliches Konzept im Sinne der konkreten Utopie der alten Arbeiterbewegung entwickeln, sondern bleibt retardierender Reflex der gegebenen Kapitalstrukturen.

\subsection{Die Krise als Chance? - Newe Elemente einer Gewerkschaftspolitik im Sinne einer "Logik der Arbeit«}

Die dargestellten Problemebenen einer traditionellen gewerkschaftspolitischen Defensivposition in der Restrukturierungskrise resultieren letztendlich aus der Anbindung der Gewerkschaftspolitik an die »Logik des Kapitals — eine Anbindung, mit der die deutsche Gewerkschaftsbewegung in der Nachkriegsprosperität gute Erfahrungen gemacht hatte. Aber die Restrukturierungskrise ist ja gerade dadurch charakterisiert, daß die alten Strukturen, die eine erfolgreiche gewerkschaftliche Lohnpolitik ermöglicht haben, aufgekündigt bzw. zersetzt werden. Wenn die Gewerkschaften in der gegenwärtigen Phase so in Gefahr ger aten, zu einem Zersetzungsprodukt der Restrukturierung des Kapitals zu werden - zwar relativ erfolgreich auf einzelnen Teilarbeitsmärkten, aber auf Kosten solidarischer Politikelemente und mit nachlassender gesellschaftlicher Relevanz -, dann steht die Frage an, ob es jenseits einer auf den status quo bezogenen Defensivpolitik Möglichkeiten gibt, neue Formen einer offensiven Politik zu entwickeln.

Diese Frage soll hier nicht hypothetisch gestellt werden. Vielmehr soll anhand der existierenden Problemlagen und gewerkschaftlichen Antworten darauf untersucht werden, inwieweit sich solche neuen Formen bereits in der Restrukturierungskrise ent wickeln, und es soll darüber hinaus danach gefragt werden, inwieweit das Kapital selbst widersprüchliche Antworten bereit hält, die Ansatzpunkte für eine neue Politik sein könnten. Es muß auch hervorgehoben werden, daß sich solche neuen Formen nur auf Basis einer Defensivposition entwickeln können, die die Aufgabe hat, kurzfristig die Folgen der Krise abzuwehren und Zeit für die Entwicklung eigener Formen gewerkschaftlicher Politik der Beeinflussung des Restrukturierungsprozesses zu gewinnen. 
Damit ist zugleich der generelle Ansatzpunkt benannt: Wenn die gegenwärtige Phase eine Phase umfassender Restrukturierung, Neuzusammensetzung der ökonomischen, sozialen und politischen Elemente der Reproduktion ist, dann kommt es darauf an, diese Neuzusammensetzung mitzubestimmen! Kriterium einer solchen »Mitbestimmung « kann dabei nicht nur die kurzfristige Durchsetzung der materiellen Interessen der gegenwärtigen Mitglieder sein, sondern muß darüber hinaus die Frage nach der zukünftigen Basis gewerkschaftlicher Organisations- und Konfliktfähigkeit sein, die Frage nach den Kommunikationsstrukturen in Betrieb, Branche, Sektor und in der Nicht-Arbeitszeit. Es geht, um eine griffige und bekannte Formulierung von Bruno Trentin abzuwandeln, um die Frage nach dem »Was, wie und für wen produzieren und wie leben?" Grundsätzlich kann dabei die Fragestellung auf drei Ebenen entwickelt werden:

1. Welche Entwicklungen ökonomisch-technologischer und politischer Art müssen verhindert werden?

2. Welche dieser Entwicklungen müssen und können beeinflußt werden?

3. Wo müssen und können die Gewerkschaften eigene, jenseits der vom Kapital vorgebenen Entwicklungslinien fordern bzw. durchsetzen?

$z u$ (1.): Hier beschränken sich die Gewerkschaften bislang weitgehend auf zwei Aspekte: Verhinderung des Abbaus der sozialen Sicherung und des politisch fixierten Systems der sozialen Beziehungen, und Verhinderung bzw. zeitliche Verzögerung des Durchschlagens technologischer Innovationen auf die soziale Situation des Lohnabhängigen (Rationalisierungsschutzabkommen, Sicherungen gegen Abgruppierungen etc.). Beide Zielsetzungen müssen sich mit Resultaten kapitalistischer Akkumulation auseinandersetzen und stehen so immer unter dem Druck des »Sachzwangs«. Insofern verweist dieses Problem schon auf die zweite Themenebene. Darüber hinaus ist aber zu fragen, ob es gewerkschaftspolitisch nicht möglich ist, bestimmte Entwïklungen ökonomischer und technologischer Art, deren Auswirkungen die gewerkschaftliche Basis in der Zukunft zerstören (z.B. durch Vernichtung von gesellschaftlich sinnvollen Arbeitsplätzen) und/oder zukünftige Interessenstrukturen produzieren, die einer solidarischen Gewerkschaftspolitik (und Gesellschaft!) entgegenstehen, zu verhindern! (Vergangene Beispiele: Akkumulation des Kapitals in der Atom- und Rüstungsbranche, aktuell: der Ausbau der Verkabelung mit überhaupt noch nicht überschaubaren sozialen und arbeitspolitischen Folgen).

zu (2.): Die Ansatzpunkte, die sich hierzu abzeichnen, sind in mittlerer Frist sicherlich die erfolgversprechendsten, handelt es sich doch darum, Tendenzen, die das Kapital selbst freisetzt, aufzugreifen und im Sinne einer Rekonstruktion gewerkschaftlicher Hegemonie auszubauen. Hier haben sich gerade in der Politik einiger DGB-Gewerkschaften unter dem Druck der Restrukturierungskrise konkrete Veränderungen der Politik herausgebildet, die im folgenden skizziert werden sollen:

- Mit ihrer Politik der Verkürzung der Wochenarbeitszeit greifen DGB-Gewerkschaften die historische Tendenz des Kapitals (wieder) auf, die notwendige Arbeitszeit zu verkürzen mit den kapitalistischen Resultaten einer wachsenden Masse von Arbeitslosen. Im Gegensatz zur Politik der Arbeitszeitverkürzung in der Prosperität (1957-1965) steht diese Politik jetzt aber von vornherein unter arbeitsmarktpolitischen Aspekten und geht somit an den unmittelbaren Interessen der beschäftigten Mitglieder vorbei und verlangt von diesen lohnpolitische Opfer. Zugleich stellt sie aber den Zusammenhang von Freisetzungen, Lohnpolitik und Nicht-Arbeirszeit her und geht so über einzelinteressen-bezogene Politikansätze hinaus in Richtung einer offensiven solidarischen Arbeitspolitik. Auch wenn das nach harten Streik- 
auseinandersetzungen erreichte Ergebnis (38,5 Stunden Wochenarbeitszeit im Durchschnitt) relativ wenig arbeitsmarktpolitische Wirkungen zeigen dürfte, so ist durch die Diskussion um diese Politik ein breites Bewußtsein zu den ökonomischen und sozialen Zusammenhängen geschaffen worden; und selbst die zunächst als Kompromiß hingenommene »Flexibilisierungsklausel« hat dazu geführt, daß durch den Zwang der betrieblichen Aushandlung die Auseinandersetzungen um die Forderungen betriebsnah fortgeführt wurden und dort erneut mobilisierten.

- Rationalisierungsschutzabkommen, Lohnrahmentarifverträge, Innovationsberatungsstellen, Mitbestimmungsformen (am Arbeitsplatz, im Betrieb, auf Konzernebene) können Ausgangspunkte einer umfassenderen »Produktionspolitik« sein, die die Gewerkschaften befähigt, technologische Entwicklungslinien und deren konkrete Anwendungen zu kontrollieren. Dabei geht es darum, die Zerstörung von sozialer Kommunikation, die Verfeinerung von betrieblicher Kontrolle und den Verlust der bislang verbliebenen Souveränität der Lohnabhängigen über ihre Zeit zu verhindern. Darüber hinaus kann aber auch an positive Tendenzen in den neuen Techniklinien angeknüpft werden bzw. diese müssen u.U. gegen das Management und das Kapital durchgesetzt werden: So zeigen etwa Kern/Schumann anhand der »neuen Produktionskonzepte«, daß das Kapital selbst ein Interesse daran haben kann, den Arbeitsplatz bestimmter Facharbeitergruppen zu requalifizieren, der Facharbeit - in Abkehr von tayloristischen Konzepten - einen erneuerten autonomen Spielraum zu geben (vgl. Kern/Schumann 1984 - kritisch Düll 1985, Malsch 1985). Gewerkschaftspolitisch käme es hier - neben der Vertretung der Interessen der mehrheitlichen Rationalisierungsverlierer im Zuge der Durchsetzung dieser »neuen Produktionskonzepte «! - darauf an, diesen Spielraum auch zu nutzen und zu verhindern, daß sich daraus Sonderinteressen einzelner privilegierter Facharbeitergruppen gegen eine solidarische Gewerkschaftspolitik entwickeln.

- Die Entwicklung der Beschäftigung zum Dienstleistungssektor hin muß von den Gewerkschaften organisatorisch aufgefangen werden. Dieser Bereich ist aber aufgrund seiner Struktur (Klein- und Mittelbetriebe, hoher Angestelltenanteil, hoher Anteil der technischen und betriebswirtschaftlichen Intelligenz) nicht einfach nach den Mustern des traditionellen $\gg \mathrm{Ma}$ schinenarbeiters« zu organisieren. Wollen die Gewerkschaften aber hier korporatistische Interessenvertretungsstrukturen verhindern bzw. in der Konkurrenz mit Angestelltengewerkschaften (DAG etc.) bestehen, dann muß ihre Politik stärker auf die soziale Form der Arbeit (in einem sozialen Herrschaftsverhältnis befindlichen Arbeit) abzielen. Denn der»Weg in die Dienstleistungsgesellschaft « ist keineswegs ein Weg aus dem Kapitalismus, wohl aber ein Weg aus der Dominanz der Beschäftigung im industriellen Sektor mit seinen besonderen Formen der körperlichen Arbeit und des Produzentenstolzes, an denen sich gewerkschaftliche Politikformen noch zu eng festmachen.

- Die bis vor kurzer Zeit insbesonders von neuen sozialen Bewegungen artikulierte Ökologiefrage wird immer stärker von den Gewerkschaften aufgegriffen und als Frage nach den Belastungen durch Schadstoffe im Betrieb gewendet. Hier ergeben sich Überschneidungen mit der Ökologiebewegung, die den Zusammenhang von Verursachungsinstanz (Betrieb), Belastung der Lohnabhängigen durch Schadst offe und Belastung der Gesellschaft durch »Externalisierung « dieser Schadstoffe rekonstruieren könnten. Ebenso wie bei den Problemen der Arbeitslosigkeit und neuen Armut stellt sich hier die Frage nach einer gesellschaftlichen Lösung, die aber bereits inner- und außerhalb der Betriebe als Kampf gegen die Produktion von und mit Schadstoffen entwickelt werden kann. Auch die Ökologiefrage ist in unserem System letztlich eine Frage nach der Form der sozialen Arbeit. 
- Die Flexibilisierung in der Produktion mobilisiert individualistische, auf Selbstbestimmung und Eigenverantwortlichkeit aufbauende inhaltliche Vorstellungen von Arbeit (und Freizeit), die nicht einfach umkehrbar sind, sondern berechtigte Ansprüche an die Arbeit und die Kreativität in der Arbeit ausdrücken (vgl. — bezogen auf Arbeiterjugendliche - dazu Baethge u.a. 1986, S. 78 f.). Die gewerkschaftliche Politik kann daher einerseits - wie dies ja auch bei der Teilzeitarbeit schon angegangen wird - diese Formen flexibler Produktionsarbeit rechtlich bzw. tarifrechtlich stützen, einbinden; zum anderen kann sie die Hoffnung auf inhaltliche Selbstverwirklichung in der Arbeit aufnehmen und gegen ihre Einbindung in ein kapitalistisches Herrschaftsverhältnis - die diese Hoffnungen immer wieder frustriert wenden!

$Z u$ (3.): Mit den sichtbar negativen Folgen der kapitalistischen Restrukturierungskrise besteht für die Gewerkschaften auch die Chance, die Zwecke des Wirtschaftens und cie Richtung der Akkumulation zur Diskussion zu stellen: Am deutlichsten wird die »Irrationalität« des kapitalistischen Akkumulationsprozesses in der Diskrepanz von Produktivkraftentwicklung und Massenarbeitslosigkeit, von Produktion privaten Reichtums bei Produktion von »Neuer Armut «, von Verschwendungs- und Rüstungswirtschaft bei gleichzeitiger Vernichtung von Lebenschancen durch Umweltkatastrophen. In den »Konversions-Debatten«, den Programmen Arbeit und Technik« und »Umweltschutz und qualitatives Wachstum« deuten sich solche Dimensionen an.

Letztlich muß eine »soziale Beherrschung « der ökonomischen und der darin enthaltenen technologischen Entwicklung - wie sie in diesen Programmen postuliert wird - aber vom Zweck der gesellschaftlichen Produktion in unserem System, der quantitativen Ausdehnung des Profits, Abstand nehmen. Der Mangel einer gesellschaftlichen Alternative jenseits des traditionellen Sozialismus-Modells kann dabei auch eine Chance (zur Neuformulierung) sein. Die gegenwärtig sich zuspitzenden, weltweiten, aber auch binnenstaatlich sich durchsetzenden gesellschaftlichen und ökologischen Probleme verlangen nach einer bewußt gesellschaftlichen Regulierung auf der Basis einer Gebrauchswertorientierung der Produktion und der Solidarität der Produzierenden. Diese scheinbar so abstrakte Problem- und Lösungsdimension ist aber unmittelbar am Arbeitsplatz vorfindlich und aufgreifbar: Die Frage nach dem "was produzieren« wird von den Belegschaften gestelit, die aufgrund des Nichabsatzes ihrer kapitalistisch produzierten Waren auf dem Weltmarkt konkursbedingt entlassen werden. Die Frage nach dem »wie produzieren« wird im Zuge der Einführung der neuen Technologien und der Flexibilisierungskonzepte auch von den Gewerkschaften artikuliert und der "technische Fortschritt " wird angesichts der Rationalisierungsfolgen, der Dequalifizierungsprozesse und derökologischen Probleme auf seine gesellschaftliche Form hin hinterfragt. Die Frage nach dem »für wen produzieren« ist angesichts der rapiden Entwicklung der Produktivkraft der Arbeit und der Verwendung gesellschaftlichen Reichtums zum Zwecke der weltweiten Spekulation bei gleichzeitiger Verarmung und Entwicklung der Destruktivkräfte längst nicht mehr nur ein traditionalistisches Lippenbekenntnis in gewerkschaftlichen Mitgliederzeitschriften. Auf diesen Ebenen können daher - und müssen, wenn man der neokonservativen Antwort auf die kapitalistische Krise Paroli bieten will - konkrete Alternativen bzw. Strukturveränderungen im Rahmen einer »Logik der Arbeit«, der Arbeits- und Lebensbedingungen der Produzenten des gesellschaftlichen Reichtums, entwickelt werden. Dazu gehört zentral, daß die Autonomie der Kapitalbesitzer über die Verwendung des Profits eingeschränkt wird, um die spekulative Verwendung gesellschaftlichen Reichtums zu verhindern und eine gebrauchswert-orientierte Produktion in zentralen Produktionssphären ein- 
zuleiten. Dazu gehört auch eine Ausweitung des sozialen Konsums und der staatlichen Interventionen in die Ökonomie, die aber verbunden werden muß mit einer Kontrolle dieser Interventionen "von unten « - Demokratisierung also als Antwort auf die Bürokratiekritik der Neokonservativen und ihre Pseudodemokratie des Marktes.

\section{4. "Restrukturierung« der Gewerkschaften?}

Für die Gewerkschaftsorganisation ergibt sich aber auch aus einem solchen Politikbündel die Notwendigkeit einer organisatorischen und »ideologischen« Restrukturierung:

- Angesichts der vielfältigen Formen der Flexibilisierung, Dezentralisierungen und Formen der Individualisierung muß eine Organisation, in der (aus guten Gründen) eine stark zentralistische Politikform vorherrscht, den Mitgliedern gegenüber eine abstrakte Größe werden. Eine basisnahe Demokratisierung, die der Gewerkschaftsorganisation Informationen über die vielfältigen Problemanlagen und Interessen verschaffen könnte, müßte also mit der einheitlichen Politikformulierung in grundsätzlichen Fragen verbunden werden. Damit stellte sich erneut die Frage nach dem Verhältnis von Individuum und Klasse/Klassenorganisation: Wenn den Neokonservativen der individualistische Ansatzpunkt streitig gemacht werden soll, dann bleiben die traditionellen Antworten der organisatorisch-zentralistischen Einbindung des Individuums ungenügend. ${ }^{11}$

- Angesichts des Bedeutungsgewinns der Nicht-Arbeitszeit durch die Politik der Arbeitszeitverkürzung und angesichts der Dominanz des Kapitals in der Freizeit, der Durchkapitalisierung des Freizeitbereichs mit Hilfe neuer Kommunikationstechnologien und Medien, ist die erneute Ausweitung gewerkschaftlicher Arbeit über den Produktionsbereich hinaus unabdingbar - ansonsten zerstört die in der Nicht-Arbeitszeit dominierende »Individualisierung « jeden Erfolg solidarischer Politik in der Produktion.

- Nur über die Öffnung der Gewerkschaftsbewegung gegenüber existierenden gesellschaftsk ritischen sozialen Bewegungen und/oder die Reaktivierung von Elementen einer eigenen sozialen Bewegung kann die Restrukturierungskrise als Chance aufgegriffen werden und der Schwerkraft des Kapitals - der eine Politik, die sich allein auf(Teil-)Arbeitsmärkte stützt, zum Opfer fallen muß - entgegen gewirkt werden.

Die hier aufgezeigten Problemebenen der Gewerkschaftspolitik in der Restrukturierungskrise können nicht mehr in den herkömmlichen Rastern des "Verteilungskonflikts« bewältigt werden; sie resultieren aus der kapitalistischen Form des Reproduktionsprozesses, den auf die Kapitalverwertung gerichteten Zweck der Produktion. Insofern kann der Verteilungskampf auch nur ein Element der Gewerkschaftspolitik in der Restrukturierungskrise sein. Zugleich entzündet sich an vielen Punkten des Restrukturierungsprozesses (zukünftige Techniklinien, Ökologie, Dauerarbeitslosigkeit etc.) gesellschaftlicher Widerstand neben der Gewerkschaftspolitik, teilweise auch gegen sie (sofern die Gewerkschaftspolitik sich am Akkumulationsinteresse des Kapitals orientiert). Dieser Widerstand kann aber durchaus im Sinne einer Offensive gegen die marktradikalen, kapitalistischen Alternativen aufgenommen werden in einer Bündniskonzeption. Die Gewerkschaften allein wären für eine solche Aufgabe sicherlich »funktional überfordert«. Ansatzpunkte für ein solches Aufnehmen von Elementen einer neuen sozialen Bewegung sind aber durchaus in der Restrukturierungskrise vorgegeben. Die Frage ist allerdings schon, ob sich die deutschen Gewerkschaften zu einer dazu erforderlichen »neuen Kultur des Protestes« (M. Kempe) durchringen können. 
Die aufgezeigten Entwicklungsperspektiven der Gewerkschaftspolitik, für die sich konkrete Ansatzpunkte zeigen, stehen in einem diametralen Gegensatz zur neokonservativen Lösungsform der Krise. Während diese an die kurzfristigen Interessen der Arbeitnehmer als "Eigentümer der Ware Arbeitskraft« anknüpft, diese Interessen absolut setzt im Rahmen ihres Marktmodells und so die Individualität des einzelnen bestärkt (»Jeder ist seines Glückes Schmied«), liegt eine alternative Perspektive der Gewerkschaften darin, an das soziale Herrschaftsverhältnis anzuknüpfen. Nur so kann sie gegen den neokonservativen Ansatz verstärkt Solidarität und gesamtgesellschaftliche Vernunft mobilisieren. Konkret heißt dies aber, Elemente einer sozialen Bewegung hinter einer arbeitsmarktbestimmten Lohnpolitik $\mathrm{zu}$ aktivieren. Eine solche soziale Bewegung wird - wie die Auseinandersetzungen um den $\$ 116$ Anfang 1986 gezeigt haben - durch die substantiellen Angriffe der Neokonservativen auf die (tarif-)politische Kultur in der Bundesrepublik auch vom politischen Gegner provoziert. Aber diese harten Auseinandersetzungen können auch dazu führen, daß die Basis der Neokonservativen - die CDU/CSU ist ja bekanntlich zu einer populistischen Partei geworden - unterschätzt wird. Ohne eine theoretische und politische Antwort der Linken und der Gewerkschaften auf den erfolgreichen individualistischen Ansatz der Neokonservativen, dessen Erfolg durch die ökonomische und technologische Basis von Produktion und Reproduktion befördert wird, kann sich eine erneuerte gesellschaftliche Hegemonie der Gewerkschaften und der Linken schwerlich ergeben. Zumal aktuell eher einzelne soziale Bewegungen diese individualistischen Ansatzpunkte ihrerseits aufnehmen und gegen die Gewerkschaften als Repräsentanten einer "Massenkultur" und »zentralistisch-bürokratischen« Politik wenden können. Das heißt aber auch: Ohne eine Reaktivierung einer sozialen Bewegung und einer gesellschaftlichen Perspektive jenseits der vom Rentabilitätskalkül vorgezeichneten droht die Reduktion der Gewerkschaftspolitik auf eine bloße Besitzstandswahrungspolitik - ein »Besitzstand«, der über die Wirkungen des kapitalistischen Restrukturierungsprozesses kleiner wird. 


\section{Anmerkungen}

1 Die Individualisierungsthese von U. Beck (1983), die hier positiv übernommen wird, müßte allerdings noch genauer diskutiert werden. Gegen die enge Anbindung dieses Phänomens an den Wohlfahrtsstaat kann m.E. diese These auch und gerade mit der Marxschen Analyse auf die »Normalität « des kapitalistischen Produktions- und Reproduktionsprozesses zurückgeführt und begründet werden; die wohlfahrtsstaatliche Sicherung würde in dieser Sicht die angelegten Tendenzen der privatistischen Haltung und Individualisierung besonders in der Nicht-Arbeitssphäre verstärken. Auch scheint mir, daß Beck zu wenig den Marxschen Widerspruch von individueller Freiheit (in der Zirkulationssphäre) und der über den Verkauf der Ware A rbeitskraft eingeleiteten sozialen Herrschaft berücksichtigt, der eine Reduktion der Herausbildung der Klasse auf Homogenisierung als nicht hinreichend erscheinen läßt.

2 Diese »Amerikanisierungsthese« wird von R. Erd und C. Scherrer mit dem Hinweis zurückgewiesen, daß für jede Gewerkschaftsbewegung der Zusammenhang zwischen Arbeitsmarktsegmentierung und Organisationsstruktur konstitutiv sei. (1984, S. 88 f.) Dieser Einwand reduziert die Gewerkschaften auf reine Verkaufsunternehmen der »Ware Arbeitskraft « auf den Teilarbeitsmärkten, ohne die Bedeutung des dadurch eingeleiteten Herrschaftsverhältnisses für die Theorie und (eben nachlassenden!) Praxis der kontinentaleuropäischen Arbeiterbewegungen und Gewerkschaften überhaupt noch in den Blick bekommen zu können. Die Frage ist hier die, ob eine solche Reduktion, die für etliche US-Gewerkschaften sicherlich konstitutiv ist, sich durchsetzt und mit welchen Folgen.

3 Vgl. dazu Altvater u.a. 1979, Altvater u.a. 1983, SOST 1983. Die Analyse dieses Strukturbruchs unterscheidet sich dabei im Detail; gemeinsam ist diesen Analysen aber die Begründung einer Veränderung der materiellen und sozialen Basis der Reproduktion auf dem Weltmarkt, ohne auf kontingente Faktoren oder auf problematische Annahmen im Kontext der Theorie von den »Langen Wellen« zurückzugreifen.

4 Überspitzt könnte gegen eine basisdemokratische Position in der Neuen Linken damals argumentiert werden: Gerade weil die Gewerkschaft die Interessen ihrer Basis vertritt, wird sie jetzt Teil des "Atomfilzes«. Hier zeigt sich auch besonders deutlich, wie über die Kapitalakkumulation Fakten geschaffen wurden, an die Interessen gebunden waren, die eine bloß einzelinteressen-orientierte Gewerkschaftspolitik hilflos den Kapitalinteressen auslieferte. Es sollte nicht vergessen werden, daß die Arbeitskraft als »variables Kapital in der Marxschen Theorie Teil des Kapitals ist...

5 Insofern dieser Zusammenhang in der Weltwirtschaftskrise der 70er Jahre durch die Eigendynamik der Ökonomie ("strukturelle Überakkumulation«) zur Disposition gestellt wird, ist es berechtigt, im Sinne der Fordismus-These von einer Krise der Regulation« zu sprechen (vgl. Lipietz 1985, vgl. auch Esser/Hirsch 1984). Wenn ich hier dennoch die Fordismus-These nicht weiter in der Argumentation verfolge, dann deshalb, weil wesentliche Elemente dieser These sich in der deutschen Entwicklung nicht nach weisen lassen: die besondere Stellung des Maschinenbaus und auch von Teilen der Elektroindustrie gründet gerade nicht in der Massenproduktion, sondern in der Einzel- und Kleinserienproduktion und ist eng an die spezialisierte Qualifikation des Facharbeiters gebunden, die wiederum den Kern der Gewerkschaftsmitglieder stellen; die keynesianische Regulierung und der Einbau der Gewerkschaften in diese Regulierungsform erfolgt relativ spät, gegen Ende des Nachkriegsaufschwungs.

6 Vgl. dazu Hofer/Schnur 1986, S. 40 f.; der »moderne Industriebereich«, der »auf dem Höhepunkt industrieller Reife zu Beginn der 70er Jahre $37 \%$ der Erwerbbevölkerung beschärtigte, ist »seit Mitte der 70er Jahre im Rückgang begriffen«(Kleber/Stockmann 1986, S. 60).

7 Es ist in diesem Zusammenhang zu betonen, daß sich das Gewicht des Dienstleistungssektors im Beschäftigungssystem relativ verstärken wird, aufgrund des Rückgangs in den anderen Sektoren; von der Beschäftigungsentwicklung in absoluter Zahlen ist eine "Kompensationsfunktion« des Dienstleistungsbereichs nicht zu erwarten. Die Verschiebung zugunsten dieses Sektors in den 60er und 70er Jahren belegt die folgende Tabelle: 
Nach Kleber/Stockmann (1986, S. 58) ergeben sich z.B. folgende Veränderungen - Erwerbspersonen nach Wirtschaftsbereichen in Tsd.

\begin{tabular}{lccc} 
& 1950 & 1970 & 1980 \\
\hline 1. Landwirtschaft & 5451 & 2035 & 1400 \\
\hline 2. Sekundärer Sektor & & & \\
Produktionsmittel ... & 6058 & 9751 & 9055 \\
$\quad$ Verbrauchsgüter & 3834 & 3239 & 2545 \\
\hline 3. Tertiärer Sektor & & & \\
Handel, Verkehr, Geschäftl. Dienste & 3818 & 5973 & 6198 \\
Konsumptive Dienste & 1472 & 1454 & 1281 \\
Staatl./wohlfahrtsstaatl. D. & 2369 & 3885 & 5784 \\
\hline
\end{tabular}

Anstieg der Erwerbspersonen insges. 23002 (1950)

auf $26280(1980)$

8 Vgl. z.B. die Diskussion in den WSI-Mitteilungen: Schmidt, Düll, Helfert (alle 1985) und die Replik von Kern/Schumann (1985) bzw. die ähnlich gelagerte Kontroverse von Hartmann (1985) und Schmiede/v. Greiff (1985). In der Diskussion wird oft vernachlässigt, daß Kern/Schumann wie auch Baethge/Oberbeck betonen, daß ihre Untersuchungsergebnisse nicht einfach auf die gesamten ökonomischen Sektoren zu beziehen sind und die durch die Rationalisierungsverlierer dominierten Arbeitsmarktverhältnisse der Zukunft nur gesamtwirtschaftlich zu bewerten (und zu lösen) sind. In dieser Perspektive verbietet sich auch die These von Glotz (1986), der aus den neuen Produktionskonzepten »den« Arbeiter der Zukunft entstehen sieht. Zur Kritik der optimistischen Sicht bei Kern/Schumann vgl. auch Lucas 1986, eine differenzierende Sicht bei Jürgens u.a. 1985 und Malsch 1985.

9 Bezogen auf die Automobil-Industrie in der Bundesrepublik kommen z.B. Jürgens u.a. (1985, S. 24) zu folgendem Ergebnis: »Unsere These in diesem Aufsatz geht dahin, daß die Entwicklung neuer Produktionskonzepte in der Automobilindustrie der Bundesrepublik behersscht wird von der Technikstrategie. Demgegenüber liegt die Attraktivität des »Japanischen Modells« gerade im Versprechen gesteigerter Effizienz und verringerter Kosten ohne Investitionen.« Allerdings heißt dies nicht, daß nicht auch »japanische « Organisationsformen versucht werden, diese bestehen aus: »1. der Bildung von Arbeitssystemen/Arbeitsgruppen, die den Arbeitsseinsatz flexibler machen sollen; 2. der »Anreicherung« der Produktionsarbeit mit Qualitätsverantwortung, 3. der Bildung von Qualitätszirkeln und ähnlichen Gruppen« (ebd.).

10 Diese Kritik trifft gleichermaßen die Diskussion in der Linken der Bundesrepublik insgesamt, die das Verhältnis von Individuum und Klasse oder Gesellschaft stets zuungunsten des Individuums gelöst hat (bis hin zu den extremen Karikaturen der K-Gruppen und der RAF). Insofern ist die z.Zt. sich entwickelnde Diskussion um die These von der Individualisierung und den Individualismus nur zu begrüßen. Vgl. etwa Przeworski 1986.

\section{Literatur}

Altvater, E. u.a. 1979: Vom Wirtschaftswunder zur Wirtschaftskrise, Berlin

Altvater, E. 1983: Bruch und Formwandel eines Entwicklungsmodells, in: J. Hoffmann (Hrsg.), Überproduktion, Unterkonsumtion, Depression, Hamburg

Altvater, E. u.a. 1983: Alternative Wirtschaftspolitik jenseits des Keynesianismus, Opladen

Bäcker, G. 1985: Sozialpolitik im Verteilungskonflikt, in WSI-Mitteilungen Nr. 7

Baethge, M. u.a. 1986: Jugendliche auf Distanz, in: Gewerkschaftliche Monatshefte Nr. 2/

Baethge, M./Oberbeck, M. 1986: Zukunft der Angestellten, Frankfurt/M New York 
Beck, U. 1983: Jenseits von Klasse und Stand? in: R. Kreckel (Hrsg.), Soziale Ungleichheiten, Soziale Welt, Sonderband 2, Göttingen 1983

Bericht 1983: Bericht der Kommission »Zukunftsperspektiven gesellschaftlicher Entwicklungen«, Stuttgart 1983

Blanke, B. 1977: Formen und Formwandel des politischen Systems in der bürgerlichen Gesellschaft, in: V. Brandes u.a. (Hrsg.), Handbuch 5 Staat, Frankfurt/M - Köln

Bonß, W./Heinze, R.G. 1984: Arbeit, Lohnarbeit, ohne Arbeit, in: W. Bonß, R.G. Heinze(Hrsg.), Arbeitslosigkeit in der Arbeitsgesellschaft, Frankfurt/M

Buci-Glucksmann, B. 1982: Sozialdemokratie und keynesianischer Staat, in: Prokla Nr. 47

Büchtemann, Ch. 1984: Der Arbeitslosigkeitsprozeß, in: W. Bonß, R.G. Heinze (Hrsg.), Arbeitslosigkeit in der Arbeitsgesellschaft, Frankfurt/M

Dahrendorf, R.: Verhindern statt vorantreiben? in: Die Zeit v. 18.5. 1984

Dombois, R./Osterland, M. 1982: Neue Formen des flexiblen Arbeitskräfteeinsatzes: Teilzeitarbeit und Leiharbeit, in: Soziale Welt Nr. 3-4

Düll, K. 1985: Gesellschaftliche Modernisierungspolitik durch neue »Produktionskonzepte«? in: WSIMitteilungen Nr. 3

Erd, R./Scherrer, Ch. 1984: Amerikanische Gewerkschaften - Opfer des Weltmarkts, in: Prokla Nr. 54

Esser, J. 1982: Gewerkschaften in der Krise, Frankfurt/M

Esser, J./Hirsch, J. 1984: Der CDU-Staat « Ein politisches Regulierungsmodell für den »nachfordistischen« Kapitalismus, in: Prokla Nr. 56

Glotz, P.: Die Zuspitzung der Arbeit - Fünf Thesen zur Arbeitsgesellschaft und ihrer Zukunft, in: K. v. Haaren u.a. (Hrsg.), Befreiung der Arbeit, Bonn

Hartmann, M. 1985: Dequalifizierung oder Requalifizierung der Arbeit, in: Leviathan Nr. 2

Helfert, M. 1985: Chancen neuer Produktionskonzepte, in: WSI-Mitteilungen Nr. 3

Hirsch, J./Roth, R. 1980: »Modell Deutschland« und neue soziale Bewegungen, in: Prokla Nr. 40

Hofer, P./Schnur, P. 1986: Projektion des Arbeitskräftebedarfs nach Sektoren, in: MittAB 1

Hoffmann, J. 1981: Einheitsgewerkschaft oder »korporatistische Blockbildung«? in: Prokla Nr. 43

Hoffmann, J. 1982: „Das Ende der Fahnenstange«, in: Prokla Nr. 49

Hoffmann, J. (Hg.) 1983: Überproduktion, Unterkonsumtion, Depression, Hamburg 1983

Hoffmann, J. 1985: Technologiepolitik - sozial gesteuerte Innovation via Politik? in: Walter Dörhage u.a. (Hrsg.), Technik im Griff? Hamburg

Kern, M./Schumann, M. 1985: Das Ende der Arbeitsteilung, München

Kern, M./Schumann, M. 1985: Kontroverse um »neue Produktionskonzepte« - Replik, in: WSI-Mitteilungen Nr. 6

Klauder, W. u.a. 1985: Arbeitsmarktperspektiven der 80er und 90er Jahre, in: MittAB Nr. 1

Kleber, W./Stockmann, R. 1986: Wachstum und Strukturwandel des Beschäftigungssystems, in: Soziale Welt Nr. 1

Kleinknecht, A. 1984a: Innovationsschübe und Lange Wellen, in: Prokla Nr. 57

Kleinknecht, A. 1984b: Innovation Patterns in Crisis and Prosperity, Diss. Freie Universität Amsterdam, September

Kubicek, H./Rolf, A. 1985: Mikropolis, Hamburg 1985

Leibfried, St./Tennstedr, F. (Hrsg.) 1985: Politik der Armut und die Spaltung des Sozialstaats, Frankfurt/M

Lipietz, A. 1985: Akkumulation. Krisen und Auswege aus der Krise, in: Prokla Nr. 58

Lucas, R. 1986: Kein Ende der Arbeitsteilung, in: Wechselwirkung Nr. 28

Malsch, Th. 1985: „Neue Produktionskonzepte« zwischen Rationalität und Rationalisierung - Mit Kern und Schumann auf Paradigmensuche, in: IIVG discussion papers, Berlin

Mensch, Th. 1975: Das technologische Patt, Frankfurt/M

Mückenberger, U. 1985a: Die Krise des Normalarbeitsverhältnisses, in: Zeitschrift für Sozialreform Nr. 7.8 (2 Teile)

Mückenberger, U. 1985b: Die Arbeitsrechtsinitiativen der Regierungskoalition, in: Kritische Justiz Nr. 3 
Müller-Jentsch, W. 1984: Klassen-Auseinander-Setzungen, in: Prokla Nr. 54

Mooser, J. 1983: Auflösung der proletarischen Milieus, in: Soziale Welt Nr. 3

Oppolzer, A. 1986: Wenn Du arm bist, muß Du früher sterben, Hamburg

Osterland, M. u.a. 1973: Materialien zur Lebens- und Arbeitssituation der Industriearbeiter in der BRD, Frankfurt/M

Przeworski, A. 1986: Methodologischer Indivualismus als Herausforderung der marxistischen Theorie, in: Prokla Nr. 62

Piore, M./Sabel, Ch. 1985: Das Ende der Massenproduktion, Berlin

Sabel, Ch. 1986: Struktureller Wandel der Produktion und neue gewerkschaftliche Strategien, in: Prokla Nr. 62

Schmidt, R. 1985: Zu den arbeitspolitischen Chancen und Grenzen neuer Produktionskonzepte, in: WSI-Mitteilungen Nr. 3

Schmiede, R./v. Greiff, B. 1985: Industriesoziologie als positive Geschichtsphilosophie? in: Leviathan Nr. 2

Schubert, A. 1985: Die internationale Verschuldung, Frankfurt/M

Schumann, M. 1979: Encwicklungen des Arbeiterbewußtseins, in: Gewerkschaftliche Monatshefte Nr. 3 SOST 1983: Sozialistische Studiengruppen, Bundesrepublik in der Krise, Hamburg

Späth, L. 1985: Wende in der Zukunft, Reinbek

Streeck, W. 1979: Gewerkschaftsorganisation und industrielle Bezichungen, in: Gewerkschaftliche Monatshefte Nr. 11

Väth, W. 1985: Konservative Modernisierungspolitik, in: Prokla Nr. 56 